\title{
Correction to: OsbZIP71, a bZIP transcription factor, confers salinity and drought tolerance in rice
}

\author{
Citao Liu ${ }^{1} \cdot$ Bigang Mao ${ }^{3} \cdot$ Shujun $\mathrm{Ou}^{1} \cdot$ Wei Wang $^{1} \cdot$ Linchuan Liu ${ }^{1} \cdot$ Yanbin $\mathrm{Wu}^{2} \cdot$ Chengcai Chu $^{1} \cdot$ Xiping Wang $^{2}$
}

Published online: 26 June 2018

(c) Springer Nature B.V. 2018

\section{Correction to: Plant Molecular Biology (2014) 84:19-36 https://doi.org/10.1007/s11103-013-0115-3}

Due to an error in combining the figure, an incorrect version of Fig. 9e was presented in the original publication. The photograph of induced expression line IE-4 treated by PEG was accidentally duplicated from Fig. 7d. The adjusted Fig. 9e with the correct photograph of IE-4 treated with PEG is published here as well as the corrected figure legend.

The 'Materials and methods' section was also adjusted accordingly. On page 21, line 5 of the first paragraph, in 'Stress tolerance assays' should read: For the salt treatment, 14-day-old seedlings were transferred to IRRI solution containing $\mathrm{NaCl}(150 \mathrm{mM})$ for $8-9$ days, and then plants were recovered under normal conditions for another 7-9 days. For PEG treatment, 14-day-old seedlings were transferred to culture solution containing 20\% (w/v) PEG 6000 for 5-6 days and then returned to normal conditions for another 7-9 days.

In the 'Results' section, on page 28, line 3 from the bottom of the left-hand column should be corrected as 'was $35 \%$ after salt treatment for 8 days'.

The original results and conclusions are unaffected by these corrections. The authors apologize and accept responsibility for not detecting the error prior to publication. All the authors approve these corrections.
Citao Liu, Bigang Mao and Shujun Ou have contributed equally to this work.

The original article can be found online at https://doi.org/10.1007/ s11103-013-0115-3.

Chengcai Chu

ccchu@genetics.ac.cn

$\triangle$ Xiping Wang

xipingwang@hotmail.com

1 State Key Laboratory of Plant Genomics and National Center for Plant Gene Research (Beijing), Institute of Genetics and Developmental Biology, Chinese Academy of Sciences, Beijing 100101, China

2 National Center for Molecular Crop Design, Weiming Kaituo Agriculture Biotech Co., Ltd, Beijing 100085, China

3 State Key Laboratory of Hybrid Rice, Hunan Hybrid Rice Research Center, Changsha 410125, China 
(A)

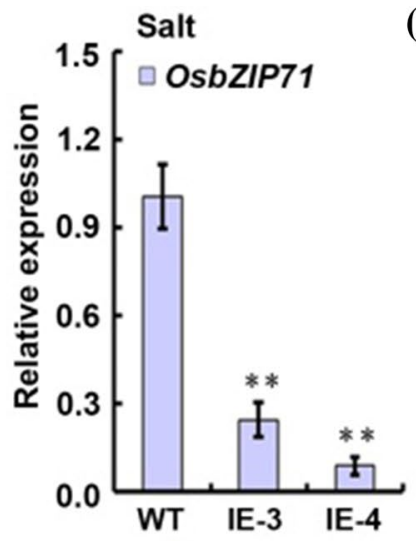

(D)

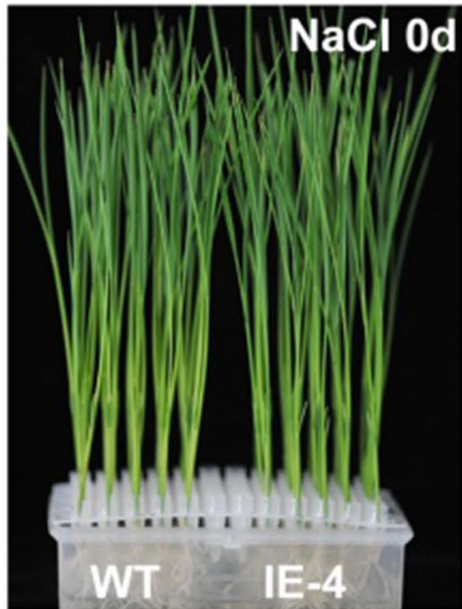

(E)

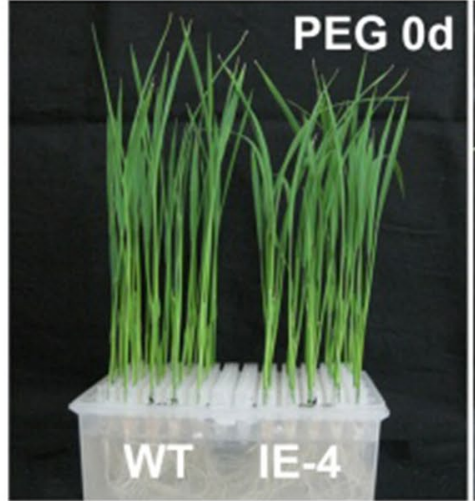

(B)
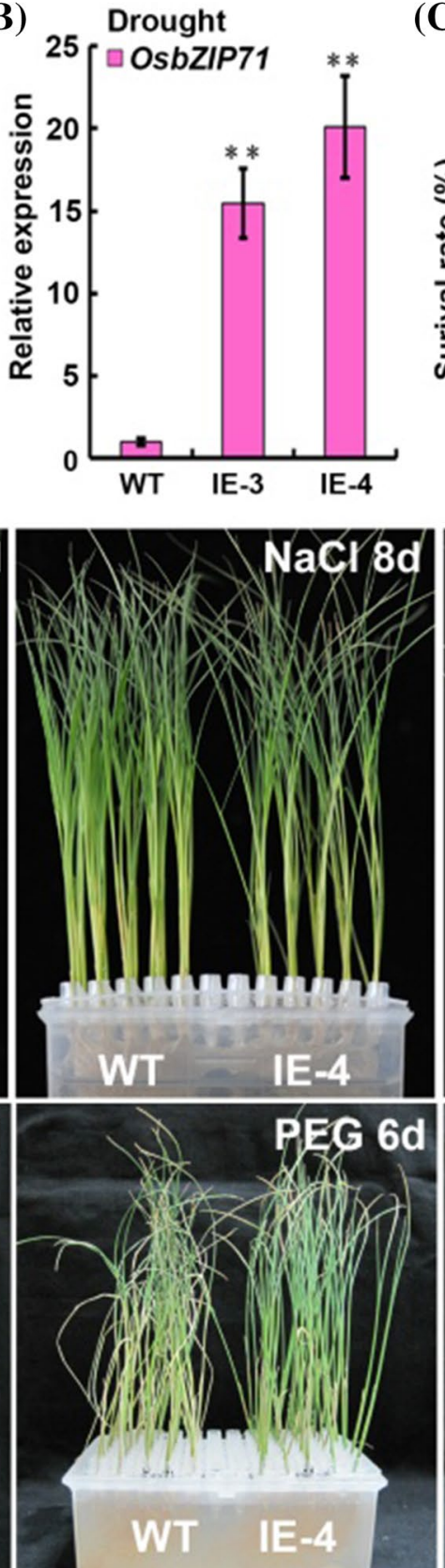
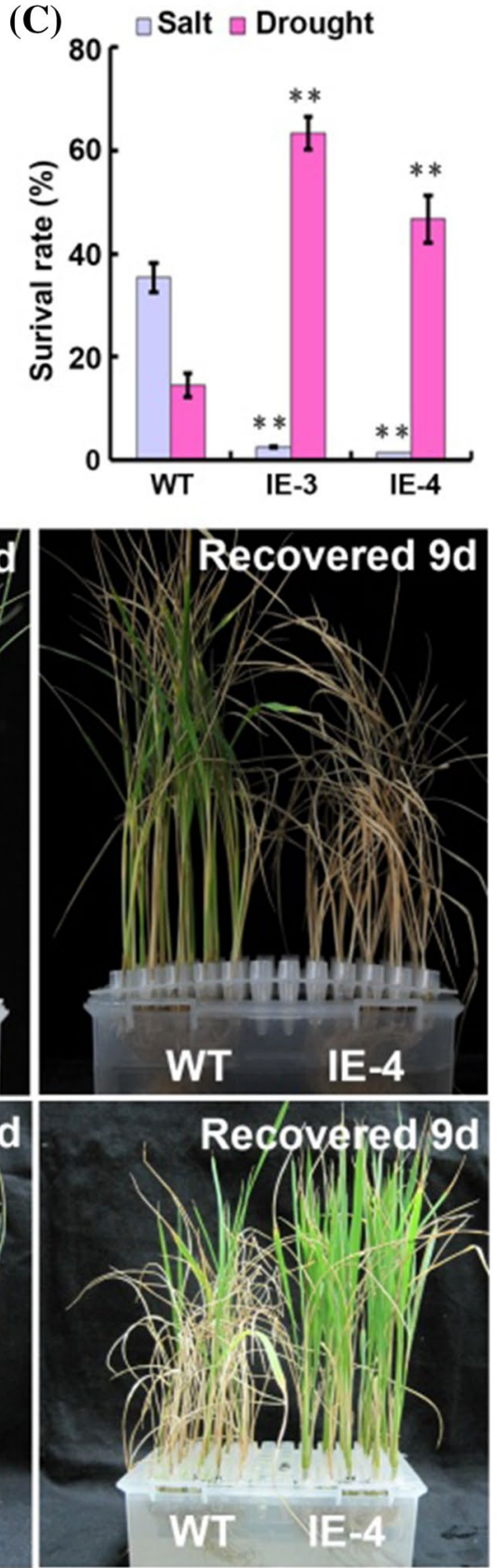

Fig. 9 Inducible expression of OsbZIP71 under the control of an inducible promoter $R D 29 A$ enhanced drought tolerance but sensitive to salt in rice. a The expression of OsbZIP71 in transgenic lines by real-time PCR analysis under drought treatment for $3 \mathrm{~h}$. b The expression of OsbZIP71 in transgenic lines by real-time PCR analysis under salt treatment for $3 \mathrm{~h}$. The error bars indicate standard deviation, values are derived from three independent biological experiments. WT wild type Zhonghua11; IE-3 and IE-4 were independent induc- ible expression lines. $\mathbf{c}$ The survival rates of wild type and transgenic lines after recovery from drought and salt treatment. All experiments were performed with three biological replicates $(\mathrm{n}=120$ each). d, e OsbZIP71 inducible expression lines exhibited more tolerance to drought stress (e) but sensitivity to salt (d) compared to wild type plants. Statistical analysis by Student's $t$-test indicated significant difference $(* * \mathrm{P}<0.01)$ 